Thorax (1954), 9, 128.

\title{
MEASUREMENT OF THE MECHANICAL FUNCTION OF THE LUNG IN NORMAL SUBJECTS
}

\author{
BY \\ JOHN L. D'SILVA AND G. KAZANTZIS \\ From the Physiology Department, London Hospital Medical College
}

(RECEIVED FOR PUBLICATION SEPTEMBER 10, 1953)

For many years the maximum breathing capacity (M.B.C.) has been used as a test of the mechanical function of the lungs. It is most commonly performed with a spirometer, which provides a permanent record of the subject's performance. Unfortunately, much of the earlier experimental work suffered from two defects. (a) No control experiments were made to investigate the fidelity with which the spirometer recorded the tidal air of the subject at high respiratory rates, and $(b)$ the rate of breathing was seldom recorded.

It has been shown (D'Silva and Mendel, 1950 ; Bernstein and Mendel, 1951 ; Bernstein, D'Silva, and Mendel, 1952) that both the above factors should be taken into account when measurements of maximum ventilatory capacity (M.V.C.) are made with the aid of a spirometer.

It is well known that the M.B.C. of a group of normal subjects may vary widely so that the deviation of an individual value from the mean may be very large. Thus, Gray, Barnum, Matheson, and Spies (1950) found a mean M.B.C. of 167.1 litres/ minute for a large group of healthy young men, but the standard deviation was \pm 21.0 litres. This large range of M.B.C. values diminishes the usefulness of this method of expressing the results of the test. D'Silva, Freeland, and Kazantzis (1953) found that in a group of normal subjects the tidal air during maximum breathing at a particular rate bore a nearly constant relationship to the vital capacity volume.

Gaensler (1951) studied the rate at which the air in the lungs (following a maximum inspiration) could be expired. He called this the "fast vital capacity" and found that, though the vital capacity volumes of normal subjects varied widely, each succeeded, on the average, in breathing out $82.7 \%$ of his vital capacity volume during the first second after starting a maximal expiratory effort. Tiffeneau, Bousser, and Drutel (1949) obtained similar results. It is well known, of course, that a patient with emphysema breathes out considerably less than $82.7 \%$ of his vital capacity volume in the first second of a fast vital capacity test. $\mathbb{N}^{\circ}$ The use of this test as a measure of the mechani- $\frac{0}{3}$ cal function of the lung has not been thoroughly investigated.

The observations made in this laboratory since $\frac{\vec{D}}{\vec{D}}$ adequate experimental precautions have been $\overrightarrow{0}$ taken to satisfy criteria $(a)$ and $(b)$ above are col- $f$ lected together and analysed in the first part of this paper. In the latter part, experiments designedo to relate the "fast vital capacity" to the M.V.C. are described and standards of normality for theseo two tests recommended.

\section{MethodS}

The subjects chosen to take part in this investigation were for the most part healthy male medical students. The results obtained from one group of young male patients attending the Out-patient $\mathrm{De}-\mathrm{F}$ partment for minor ailments unconnected with the respiratory apparatus are also included. No subject studied gave any past history of respiratory disease other than occasional "colds," and none was suffering from a "cold" at the time of his tests. The group included men of a wide range of physical proportions. They were selected only inp two senses-namely, that they were all under 3 and they all agreed to co-operate in the experio ments.

The spirometer used was that described by Bern stein and others (1952). It has been shown to b\& substantially free from recording error at the res piratory rates used. The method of conducting $\%$ test of maximum breathing has been described if detail by D'Silva and others (1953).

The fast vital capacity (F.V.C.) curve was obtained as follows:

The same spirometer was used as for the M.V.C test in conjunction with a fast-moving kymograp The speed of the drum was such that a time intep val of one second was represented by $28.5 \mathrm{~mm}$. on the paper.

The subject, standing, was asked to inspire deeply as possible, place the mask on his face, and; 
TABLE I

SWEPT FRACTIONS AT DIFFERENT RATES OF BREATHING (MEANS, RANGES, AND STANDARD DEVIATIONS)

\begin{tabular}{|c|c|c|c|c|c|c|c|c|}
\hline \multirow{2}{*}{ Group } & \multicolumn{7}{|c|}{ Swept Fractions at Respiratory Rates of } & \multirow{2}{*}{$\begin{array}{l}\text { V.C. } \\
\text { Volumes } \\
\text { (litres) }\end{array}$} \\
\hline & 20 & 30 & 40 & 50 & 60 & 70 & 80 & \\
\hline $\begin{array}{l}\text { A } \\
\text { October, } 1952 \\
16 \text { subjects } \\
\text { B } \\
\text { February, } 1953 \\
14 \text { subjects } \\
\text { October, 1951 } \\
\text { 14 subjects } \\
\text { D } \\
\text { February/March, } 1952 \\
19 \text { subjects } \\
\text { E } \\
\text { October, 1952 } \\
19 \text { subjects } \\
\text { F } \\
\text { June, 1952 } \\
16 \text { subjects } \\
\text { Mean of groups } \\
\text { A, C, D, and F }\end{array}$ & $\begin{array}{c}88.4 \\
81 \cdot 0-98 \cdot 1 \\
\pm 5 \cdot 69\end{array}$ & $\begin{array}{l}80 \cdot 0 \\
70 \cdot 8-91 \cdot 4 \\
\pm 6 \cdot 43 \\
81 \cdot 5 \\
69 \cdot 5-92 \cdot 2 \\
\pm 5 \cdot 70 \\
78 \cdot 0 \\
71 \cdot 3-90 \cdot 7 \\
\pm 5 \cdot 25 \\
79 \cdot 8 \\
55 \cdot 4-99 \cdot 2 \\
\pm 12 \cdot 3 \\
79 \cdot 6 \\
67 \cdot 8-88 \cdot 4 \\
\pm 6 \cdot 02 \\
79 \cdot 9 \\
63 \cdot 5-91 \cdot 5 \\
\pm 6 \cdot 84 \\
79 \cdot 5 \\
\pm 8 \cdot 34\end{array}$ & $\begin{array}{c}70.5 \\
63 \cdot 2-83.6 \\
\pm 6.28\end{array}$ & $\begin{array}{c}66 \cdot 4 \\
56 \cdot 3-79 \cdot 1 \\
\pm 6 \cdot 82 \\
66 \cdot 8 \\
56 \cdot 5-77 \cdot 2 \\
\pm 5 \cdot 94 \\
63 \cdot 6 \\
53 \cdot 3-81 \cdot 9 \\
\pm 8 \cdot 67\end{array}$ & $\begin{array}{c}59 \cdot 2 \\
48 \cdot 9-73 \cdot 2 \\
\pm 7 \cdot 91 \\
\\
56 \cdot 1 \\
49 \cdot 5-69 \cdot 5 \\
\pm 6 \cdot 49\end{array}$ & $\begin{array}{c}52 \cdot 9 \\
41 \cdot 2-67 \cdot 5 \\
\pm 7 \cdot 89 \\
53 \cdot 9 \\
44 \cdot 5-63 \cdot 3 \\
\pm 5 \cdot 33 \\
51 \cdot 3 \\
41 \cdot 5-64 \cdot 7 \\
\pm 6 \cdot 55\end{array}$ & $\begin{array}{l}44 \cdot 6 \\
33 \cdot 8-55 \cdot 6 \\
\pm 6 \cdot 12\end{array}$ & $\begin{array}{l}5 \cdot 08 \\
4 \cdot 23-6 \cdot 26 \\
\pm 0 \cdot 66 \\
5 \cdot 08 \\
4 \cdot 23-6 \cdot 26 \\
\pm 0 \cdot 66 \\
5 \cdot 38 \\
4 \cdot 10-6 \cdot 40 \\
\pm 0 \cdot 64 \\
5 \cdot 32 \\
3 \cdot 70-6 \cdot 78 \\
\pm 0 \cdot 80 \\
5 \cdot 24 \\
3 \cdot 76-6 \cdot 55 \\
\pm 0 \cdot 68 \\
4 \cdot 97 \\
4 \cdot 22-6 \cdot 42 \\
\pm 0 \cdot 62 \\
5 \cdot 19 \\
\pm 0.67\end{array}$ \\
\hline
\end{tabular}

on receiving a signal, to expire through the mouth into the apparatus as rapidly and as completely as possible. While expiring he was exhorted to maintain his effort until the whole volume had been expelled. This procedure was performed four times by each individual.

The tracing produced was always a smooth curve and has been called the fast vital capacity curve. The tracings were measured with a transparent perspex scale graduated horizontally in tenths of a litre and vertically in tenths of a second. The scale was placed over the tracing, and volumes expired in any particular time interval were read off directly.

In most tracings the beginning of expiration was sharply defined, and it was simple to place

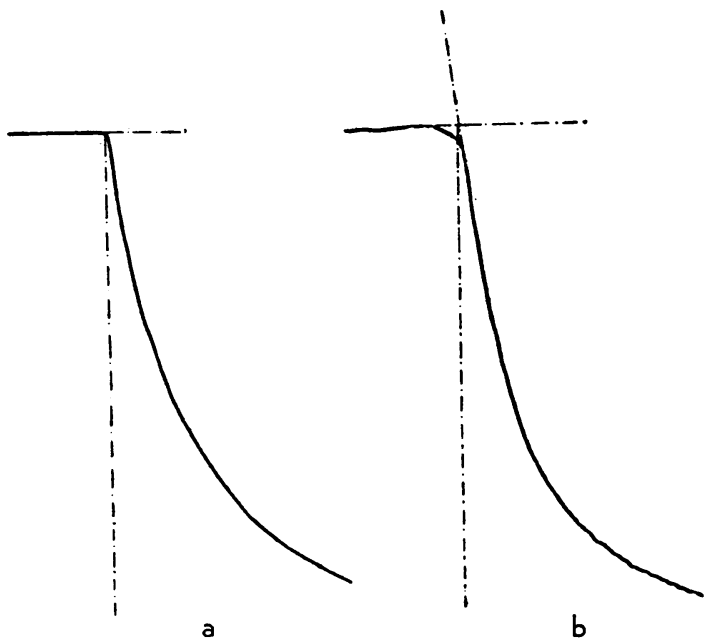

Fig. 1.-Fast vital capacity curves showing (a) sharply defined, (b) less sharply defined beginning of expiration, and the construction adopted in (b). the zero axis of the time scale (Fig. 1a). In those tracings where the start of expiration was not so clear cut, the steepest part of the curve was projected backwards and zero time was taken from the intersection of this line with the horizontal (Fig. 1b).

\section{The Maximum Breathing Capacity}

Relation between Swept Fraction during MAXIMUM BREATHING AND RATE OF BREATHING.The M.V.C. of a group of 16 medical students was determined at $20,30,50,60$, and 70 respirations per minute (R.P.M.) Each test of maximum breathing was performed once, the series of tests being preceded and followed by three determinations of vital capacity. The mean of the six determinations of vital capacity was taken as the subject's vital capacity volume. The tidal volume in each tracing was measured and the proportion of the vital capacity (V.C.) utilized in the performance of maximum breathing was calculated for each respiratory rate

$$
\text { (i.e., } \left.\frac{\text { tidal volume at } x \text { R.P.M. }}{\text { V.C. volume }} \times 100\right) \text {. }
$$

This ratio has been called the swept fraction (D'Silva and others, 1953). The results are recorded in Table I, Group A.

It will be observed that the mean swept fraction diminished as the respiratory rate increased. At each respiratory rate tested the subjects varied considerably in the proportion of the vital capacity volume which they utilized in the test, as shown by the range covered by the observations. The figures quoted for the standard deviation indicate that the scatter of the observations was much the same at all the rates of breathing tested. 


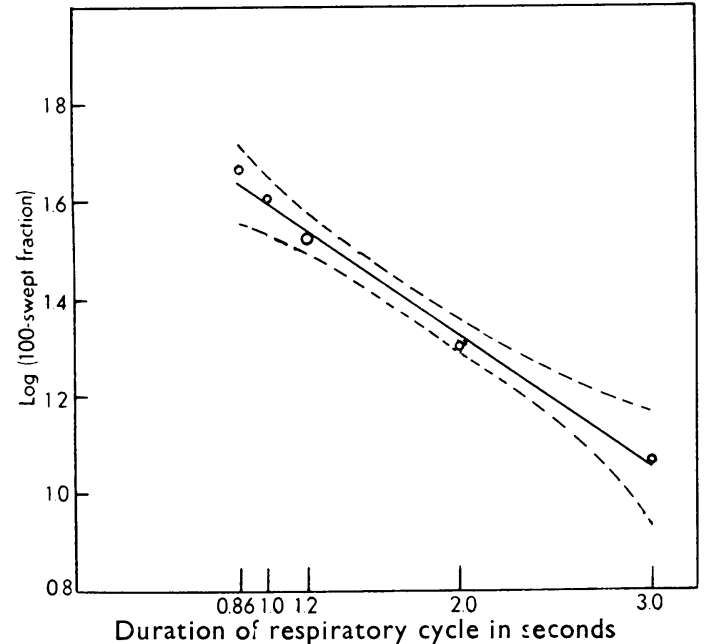

FIG. 2.-Relation between swept fraction and Iratelof breathing for subjects of Group A (Table I). are observed points; -
is the regression line. The $99^{\circ} \%$ fiducial limits are shown (- - ).

In Fig. 2 the duration of one respiratory cycle is plotted as abscissa against $\log$ (100 minus swept fraction)-i.e., $\log$ "unswept fraction"-as ordinate for the experiments in Group A. It is clear that the points obtained lie on a smooth curve, but they may also be closely represented by the straight line which has been calculated as the line of best fit. The $99 \%$ fiducial limits are shown in Fig. 2 as curved lines.

PERFormance OF Individuals at Different Rates OF BREATHING.Fig. 2 shows that the mean performances of a group of normal subjects at different respiratory rates may be regarded as being related exponentially. Fig. 3 shows the results for different subjects taken as a sample from Group A in Table I. The experimentally determined values of the "unswept" fraction, plotted semilogarithmically, have been joined by straight lines. It will be observed that of the seven curves shown, four (viz., $\mathrm{a}, \mathrm{c}$, e, and g) could be represented closely by straight lines; the other three are not such a good fit, but, in view of the fact that these curves have all been constructed from the results of a single trial at each respiratory rate, the variations from an exponential relation are probably within the limits of experimental error.
Reproducibility of the Test.-Groups B and $E$ represent duplicate determinations on subjects of Groups A and D respectively, and will be referred to again later.

Group C was a group of medical students studied by Mendel in 1951. The results of these experiments have been published previously, but in another form (Bernstein and others, 1952). They have been recalculated as "swept fractions" and included in Table I for two reasons. First, they form another group similar to $\mathrm{A}$ and, secondly, the observations were made by Mendel, whereas all the other observations recorded in Table I were made by one of us (G. K.). It will be seen that the subjects of Groups $C$ and $A$ gave very similar results at each respiratory rate tested in spite of the tests having been conducted by two different observers.

Group D was also a group of medical students whose performance in the test was studied only at 30 R.P.M. Group F, studied only at 30 R.P.M.

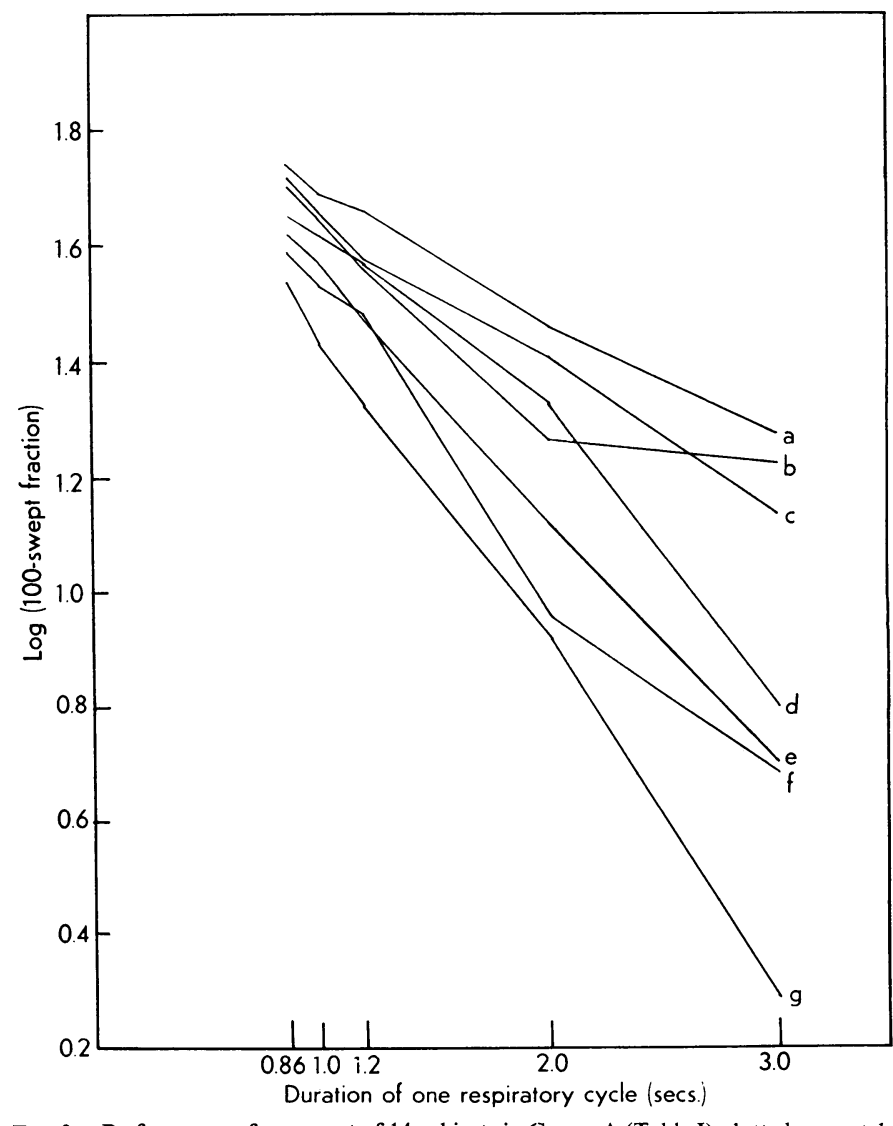

FIG. 3.-Performance of seven out of 14 subjects in Group A (Table I) plotted separately at $20,30,50,60$, and 70 R.P.M. 
was a group of patients attending the hospital for minor ailments which had no connexion with the lungs or thoracic cage.

The performance of the various groups of subjects at 30 R.P.M. was remarkably uniform. In view of the possibility that medical students might be expected to carry out this test more successfully than subjects attending an out-patient clinic, the performance of Groups $\mathrm{A}$ and $\mathrm{F}$ was compared statistically. The result $(t=0.043 ;$ d.f. $=30$; $P>0.9$ ) shows that any difference was not significant.

The test needs considerable co-operation on the part of the subject. It is therefore impracticable to perform it more than a few times on one subject in any one day. The subjects referred to in Table I breathed only once at each respiratory rate except in a few instances where the tracing showed clear evidence of a leak between the subject's face and the mask of the apparatus. In these instances the test was repeated, and if the second record showed no signs of leaks the measurement from it was included in Table I.

Though the total number of observations recorded in column 3 of Table $I$ is considerable, the mean values, the scatter, and the standard deviations recorded for the different groups of subjects are similar. This indicates that the test can be satisfactorily carried out by many individuals.

It has already been shown (D'Silva and others, 1953) that when two tests of maximum breathing are conducted at 30 R.P.M. with a short rest period between them the results agree closely. McKerrow (1953) has found similar close agreement between the results of successive determinations of M.B.C.

In Table I, Groups B and E are repeat observations on Groups A and B respectively. Two subjects originally included in Group $A$ were not tested in Group B as, at the time of the tests, they were suffering from "colds." The mean performance of the subjects of Group B was the same at the rates of breathing tested as their performance four months previously. In Group D there were two subjects who considerably improved their performance when tested seven months later. This had the effect of narrowing the range within which the observations fell and accounts for the smaller standard deviation in Group E than in Group D.

Fig. 4 is a graphical representation of the individual differences between the swept fractions on the two occasions. The negative and positive differences are equally numerous and are scattered symmetrically about zero. This strongly suggests that the differences are the results of random errors in performing the experiments. The mean differ- ences between the swept fractions determined on two separate occasions at 30, 50, and 70 R.P.M. were $5.0,3.9$, and $4.3 \%$ respectively. The test, therefore, appears to be reproducible when performed in a normal subject twice on one day or once on different days.

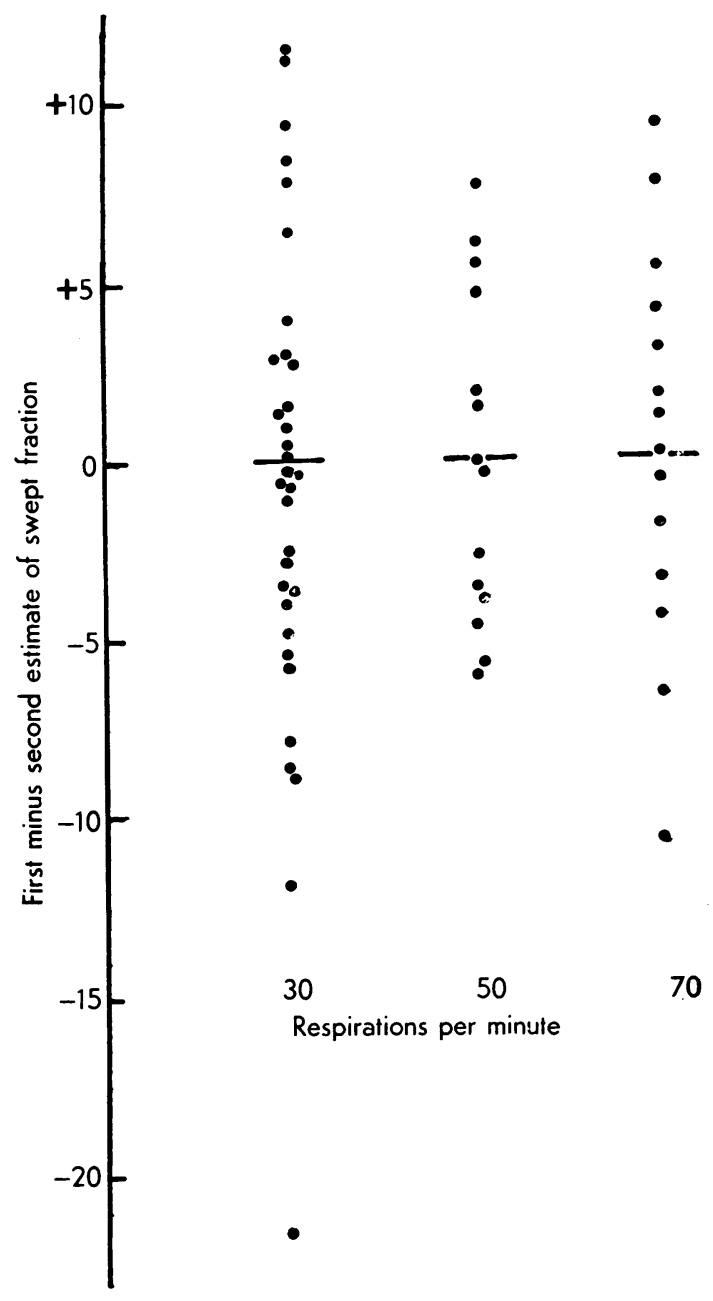

FIG. 4.-Reproducibility of M.V.C. determinations at 30, 50, and 70 R.P.M. after an interval of several months. At 30 R.P.M., observations were made on Groups $\mathbf{A}$ and $\mathbf{B}$, and $\mathbf{D}$ and $\mathbf{E}$; at 50 and 70 R.P.M observations were made on Groups $A$ and at 5 and $B$. In each case the difference plotted. Mean differences at 30,50, and 70 R.P.M. were, respectively, $5.0,3.9$, and $4.3 \%$.

Limits OF NoRMality at Different RespiraTORY RATES.-Table I gives, for each group of experiments, a measure of the scatter of the observations. However, for the calculation of the standard deviation to be valid, it is necessary to demonstrate that the observations at a given respiratory 
rate are normally distributed. This demonstration is more particularly necessary in these experiments because at 30 R.P.M., for instance, where the mean swept fraction is about $80 \%$, it is clear that there is less room for deviation on the high than on the low side of the mean.

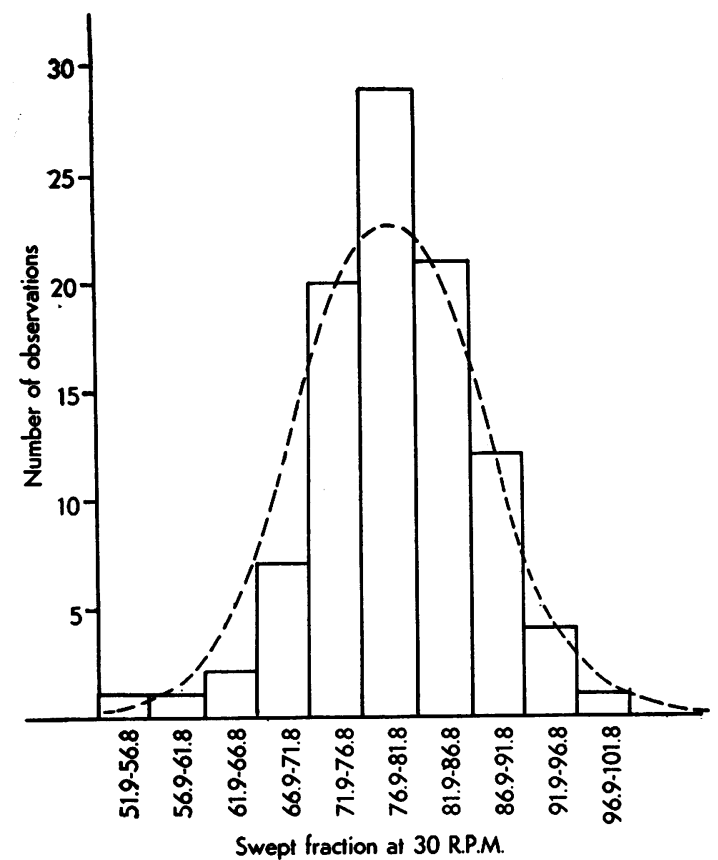

Fig. 5.-Frequency distribution of swept fractions at 30 R.P.M. about the grand mean of $79.4 \%$. The appropriate normal frequency distribution about this mean is also shown.

Fig. 5 is a histogram of all the results quoted in Table I, column 3. A respiratory rate of 30 has been chosen, not only because of the point made in the previous paragraph but also because the observations at this rate of breathing were most numerous. The difference between the distribution of the observations in Fig. 5 and the appropriate "normal distribution" was tested by the $\chi^{2}$ test and found not to be significant $\left(\chi^{2}=6.96\right.$; d.f. $10 ; 0.8>P>0.7$.

\section{The Fast Vital Capacity Curve}

Total Volume of AIr ExPIRed in F.V.C. Test. - Measurements were made of the total volume of air breathed out by each subject in a F.V.C. test. The mean volume expired in four trials is referred to as the F.V.C. volume, the mean of which (for 21 subjects) was 1.01 times (range 0.92 to 1.08 times) the volume recorded in the usual slow determination of the vital capacity.
The Reproducibility of the F.V.C. Curve.The volume of air breathed out after $1,0.6$, and 0.43 seconds was measured on each of four F.V.C. curves produced by each of 21 subjects. The mean of each group of four measurements was calculated, as was the mean deviation $(Y)$ of the individual measurements from the mean. The mean of the values of $Y$ thus obtained is an indication of the reproducibility of the F.V.C. curve. For the three time intervals referred to above, the mean value of $Y$ was $0.06,0.07$, and 0.05 litres, each of which was about $2 \%$ of the volume of air expired.

The Shape of The F.V.C. Curve.-There is a close similarity in the F.V.C. curves produced by different normal subjects. They are always smooth curves, concave upwards, but they differ in different subjects in the time taken to expel the fast vital capacity volume. This difference is small as between one normal subject and another, but in emphysema, for example, the time taken to expel the F.V.C. volume may be several times longer than normal.

Kennedy (1953) and Hirdes and van Veen (1952) state that the first part of the F.V.C. curve is a straight line. This distortion of the F.V.C. curve has been shown (Bernstein, 1954) to be due to a recording error.

The normal F.V.C. curves were at first thought to be exponential in form, differing from one another only in magnitude and time scale. Fig. 6 shows the curve representing the mean of 84 F.V.C. curves from 21 subjects. The point $A$ was chosen on the mean F.V.C. curve so that at this point $36.8 \%$ of the F.V.C. volume remained to be expired. The exponential curve decreasing to $\frac{1}{e}$ (i.e., $36.8 \%$ ) of its initial value in the same time is shown in the figure. The abscissa of the point $A$ is the time constant of the exponential curve, whose value was 0.47 seconds. From Fig. 6 it can be seen that the first part of the mean F.V.C. curve can be approximated to this exponential curve, when it can also be said to have a time constant of 0.47 seconds. The standard deviation of the individual time constants in the group of 21 observations was 0.09 seconds.

The F.V.C. curve of each subject has similarly been compared with an exponential curve and the two have always been found to be closely similar. Because of this, and the ease and accuracy with which a subject could reproduce his F.V.C. curve, it was decided to try to obtain a mathematical expression to express the average F.V.C. curve of a group of normal subjects. 


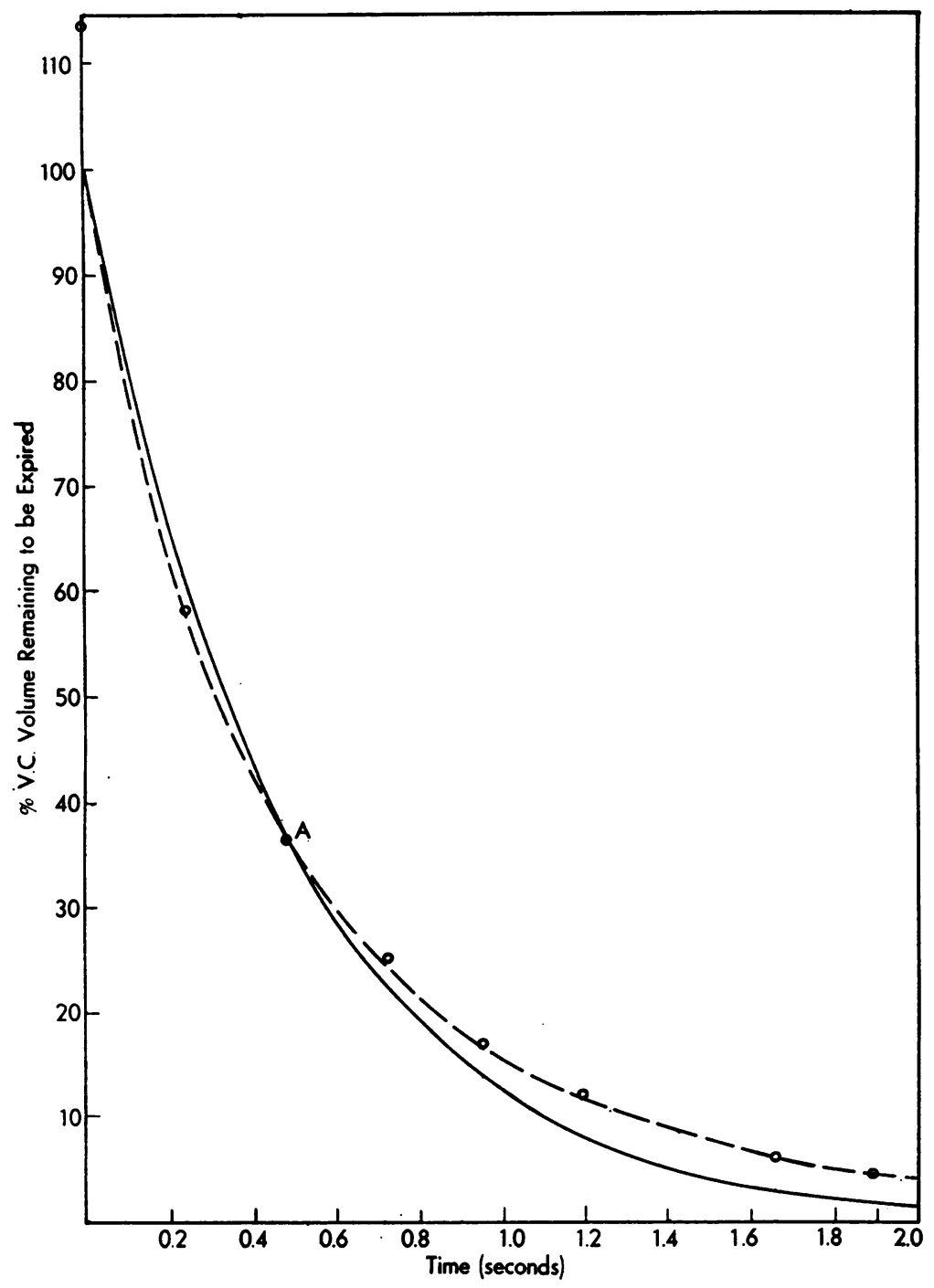

FIo. 6.-Comparison of the mean of the fast vital capacity curves of 21 subjects (- - -) with an exponential curve (- - . The plot (open circles) of the equation for an appropriate hyperbola is also shown.

It was found that a very close fit was obtained by using an equation for a hyperbola of the form

$$
y=\frac{x}{a+b x}+c
$$

which, after calculating the constants $a, b$, and $c$, became

$$
y=113.1-\frac{x}{0.0024+0.0079 x}(\mathrm{i}),
$$

where $y$ represents the percentage of the V.C. volume which remains to be expired and $x$ is the time in seconds.
The points on the curve corresponding to this equation have been plotted in Fig. 6 for different values of $x$ The close fit of the calculated points and the curve determined experimentally is apparent. An equation of type (i) was shown (Johnson, 1952) to fit the average F.V.C. curve obtained from each subject.

In order to compare the set of F.V.C. curves obtained in this group of subjects with those investigated by Gaensler (1951) and Tiffeneau and others (1949), the proportion of the F.V.C. volume expired in one second was measured. This was found to be $82.9 \%$ (S.D. \pm 4.2 ), a figure which compares well with that, $82.7 \%$, obtained by Gaensler.

THE Relationship BeTWEen M.V.C. AND F.V.C.-It is apparent from the high degree of reproducibility of F.V.C. curves and M.V.C. determinations that the individual expirations in an M.V.C. test are likely to be curves which are portions of F.V.C. curves. Kennedy (1953) found a very high correlation between the M.B.C. determined directly, and that calculated from suitable measurements of the F.V.C. curve. We have found a similar high correlation $(r= \pm 0.93)$ between the M.V.C. at 30 R.P.M. and the volume of air breathed out in 1 second during a F.V.C. test. Similarly the M.V.C. figures at 50 and at 70 R.P.M. correlate well $(\mathrm{r}=$ +0.90 and +0.85 , respectively) with F.V.C. fractions breathed out in 0.6 and 0.43 seconds, respectively, each of which is half the duration of a respiratory cycle.

Bernstein and others (1952) showed that when performing maximum voluntary ventilation normal subjects do not inflate their lungs to their fullest extent except at the lowest respiratory rates used. This experiment has been repeated on the present group of 21 subjects with similar results. 
Thus, at 20 R.P.M. the lungs are maximally inflated during each breath of the M.V.C. test, but at 70 R.P.M. the lungs are, on the average, inflated to a level which corresponds to $90 \%$ of the vital capacity volume. To test whether this factor influenced the values of $r$, the following experiments were performed.

For each respiratory rate studied the subject was asked to make an ordinary record of his vital capacity volume and, immediately afterwards, without removing his face from the mask of the spirometer, to perform maximum ventilation at a known respiratory rate set by a metronome. In this way, the relation between the subject's tidal air and his vital capacity volume could be seen and measured. A new series of correlations was calculated in which M.V.C. determinations at 30, 50, and 70

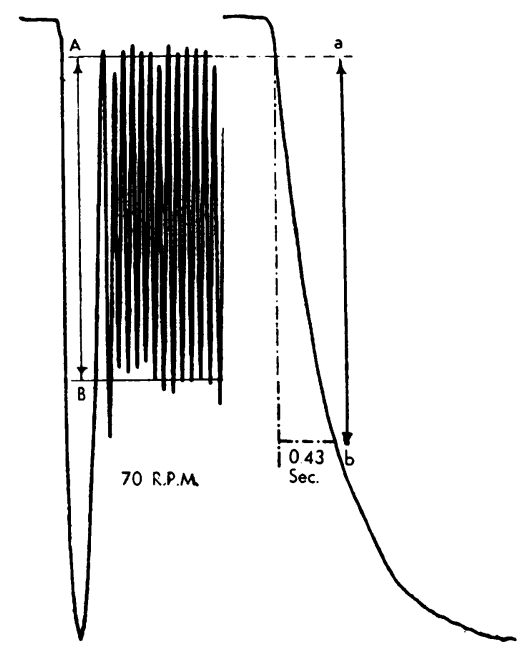

FIG. 7.-Measurement of fast vital capacity curve from the point at which expiration started in the M.V.C. test. AB is the tidal air correlated with the volume, ab, expired in the F.V.C. test.

R.P.M. were correlated with the volumes of air expired in fast vital capacity tests during 1, 0.6 , and 0.43 seconds, the measurements being made in each case not from the point of origin of the F.V.C. curve but from the point (determined above) on the curve from which expiration started in the M.V.C. test. This is illustrated in Fig. 7. The M.V.C. calculated from the tidal air AB is correlated with the volume, ab, of air expired in the F.V.C. test.

The correlation coefficients were about the same, namely, $+0.95,+0.90$, and +0.82 respectively, as those calculated when the measurements were made from the point on the curve of maximum inspiration.

\section{Discussion}

The assessment of the mechanical function of the lung has hitherto been most unsatisfactory. It $\underline{\overline{\underline{\sigma}}}$ has always been possible to obtain evidence of $\frac{\bar{\rho}}{\widehat{\sigma}}$ gross impairment of mechanical function, but $\stackrel{\mathbb{Q}}{\circ}$ minor degrees have escaped detection because of the crudeness of the experimental methods used. -

It has been the practice of observers in the past: to instruct the subject about to perform an M.V.C. $\vec{\omega}$ test to "breathe as fast and as deeply" as he was? able. This instruction appears to give the subject a free choice of rate and depth of breathing, butco in practice this is not so. The subject was nearly? always exhorted to greater effort in the sense of $\vec{\infty}^{\circ}$ increasing his rate or depth of breathing, or both.을 The form of the exhortation depended on the pre-? vious experience and judgment of the observer.c A subject instructed to breathe as fast and as deeply as possible and left to perform the test ${ }^{\Phi}$ without further instructions may choose widely different respiratory rates in successive tests. Once. ${ }^{+}$ the factor of "exhortation" enters into the deter-응 mination of maximum voluntary ventilation, the idea that the rate and depth of breathing are chosen by the subject is no longer correct.

By controlling the rate of breathing and by using $\frac{\varnothing}{\varnothing}$ a suitably constructed apparatus, the unswept frac $-\overrightarrow{\vec{\theta}}$ tion (i.e., 100 minus swept fraction) has been shown to be inversely related in an approximately exponential manner with the duration of a respiratoryo cycle (cf. Fig. 2 ; Cara and Economides, 1952). We have observed, in preliminary experiments, thato patients with marked emphysema usually show a linear relationship between the swept (or unswept) fraction and the rate of breathing, but we have not. as yet investigated cases of early emphysema, be-ô cause there is always so much doubt about the diagnosis.

To investigate in detail the relationship between the unswept fraction and the rate of breathing in individual patients would be a time-consuming procedure and very exhausting for the patient. We have found it more satisfactory to estimate the subject's performance at each of three respiratory rates (duplicate determinations) and compare them with the standard "normal" figures determine by the observer making the tests. Our mean figures are shown in Table I.

The measurement of a subject's performance int the F.V.C. test shows more promise than the M.V.C. test of affording an estimate of the mech $\mathbb{\mathbb { D }}$ anical function of his lungs. There is no doub that, as stated by Kennedy (1953), this test requires less sustained co-operation from the subject and can be applied to ill patients. 
To compare fast vital capacity curves from normal subjects the first (fast) portion may be assumed to be exponential in form. It is then simple to describe the exponential curve to which the first portion of the F.V.C. curve is so closely similar by measuring its time constant. We find that the time constants of successive curves obtained from one subject are closely repeatable and that the scatter in the observations made on different normal subjects is small. This measurement, therefore, affords a means of comparing the performance of an individual with a "normal" value determined by the observer.

It cannot be too strongly urged that each laboratory should determine its values for "normal" subjects because neither the test of maximum ventilatory capacity nor the fast vital capacity test is performed entirely independently of the observer.

From our experiments we have obtained the " normal" mean values listed below.

\begin{tabular}{c|c|c}
\hline $\begin{array}{c}\text { Rate of } \\
\text { Breathing }\end{array}$ & $\begin{array}{c}\text { Mean Sweot Fraction } \\
\text { (No. of Subjects in } \\
\text { Brackets) }\end{array}$ & $\begin{array}{c}\text { Standard } \\
\text { Deviation }\end{array}$ \\
\hline 20 & $\begin{array}{c}88.4(16) \\
79.5(65)\end{array}$ & \pm 5.69 \\
30 & $70.5(14)$ & \pm 8.34 \\
40 & $65.1(30)$ & \pm 6.28 \\
50 & $57.7(30)$ & \pm 7.76 \\
60 & $52.2(30)$ & \pm 7.34 \\
70 & $44.6(14)$ & \pm 7.22 \\
80 & \pm 6.12 \\
\hline
\end{tabular}

Mean time taken to exoel all but $36.8 \%$ of the F.V.C. volume $=$ 0.47 seconds (S.D. $=009$ seconds).

Mean percentage of F.V.C. volume expired in 1 second $=82.9 \%$ (S.D. $= \pm 4 \cdot 2 \%$ ).

There is a high correlation between the tidal volume at 30,50 , and 70 breaths per minute and the volumes of air expelled during $1,0.6$, and 0.43 seconds respectively after starting a maximal expiration (cf. Kennedy, 1953). There is no theoretical justification, at present, for making use of this relationship to calculate the M.V.C., as it is known that the fast expiratory and fast inspiratory curves (both of which are involved in performing the M.V.C. test) are completely different in shape and in their time relations.

\section{SUMMARY}

The swept fraction of the vital capacity volume of normal young adults at rates of breathing between 20 and 80 respirations per minute has been measured. The unswept fraction is related approximately exponentially to the duration of a respiratory cycle.

Limits are set for the mean of a group of normal subjects and individual normal subjects at seven different respiratory rates.

The fast vital capacity curve in normal subjects has been shown to be a hyperbola which is closely resembled by an exponential curve the time constant of which is 0.47 seconds (S.D. $= \pm 0.09$ seconds).

The use of these measurements in assessing the normality or otherwise of a subject being tested is discussed.

We are indebted to Dr. L. Bernstein for his advice during the course of this work.

\section{REFERENCES}

Bernstein, L. (1954). Thorax, 9, 63.

- and Mendel, D. (1951). Ibid., 6, 297.

— D'Silva, J. L. and Mendel, D. (1952). Ibid, 7, 255

Cara, M., and Economides, E. (1952). C. R. Soc. Biol., Paris, 146,

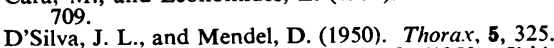

D'Silva, J. L., and Mendel, D. (1950). Thorax, 5, 325. 303.

Gaensler, E. A. (1951). Amer. Rev. Tuberc., 64, 256.

Gray, J. S., Barnum, D. R., Matheson, H. W., and Spies, S. N. (1950). J. clin. Invest., $29,677$.

Hirdes, J. J., and Veen, G. van (1952). Acta tuberc. scand., 26, 264

Kennedy, M. C. S. (1953). Thorax, 8, 73.

Johnson, J. H. (1952). Nomography and Empirical Equations, 1st ed., p. 128. Chapman and Hall, London.

McKerrow, C. B. (1953). Proc. roy. Soc. Med., 46, 532.

Tiffene a R., Bousser, J., and Drutel, P. (1949). Paris mid. (partie méd), 39, 543. 